\title{
Prontitud coordinativa: perfiles multivariados en función de la edad, sexo y estatus socio-económico
}

\author{
Alcibiades B. Valdivia ${ }^{1,2}$ \\ Rita F. Lara ${ }^{1}$ \\ Celinda B. Espinoza ${ }^{1}$ \\ Severo Q. Pomahuacre ${ }^{1}$ \\ Giovanny R. Ramos ${ }^{1}$ \\ André Seabra ${ }^{2}$ \\ Rui Garganta ${ }^{2}$ \\ José Maia ${ }^{2}$ \\ https://doi.org/10.5628/rpcd.08.01.34
}

\author{
${ }^{1}$ Universidad Nacional de Educación \\ "Enrique Guzmán y Valle" \\ La Cantuta \\ Lima \\ Perú \\ ${ }^{2}$ Laboratório de Cineantropometria e Estatística Aplicada \\ Faculdade de Desporto \\ Universidade do Porto \\ Portugal
}

\section{RESUMEN}

Objetivo: Caracterizar el nivel de desarrollo coordinativo (CoM), describir su distribución percentílica, e identificar la prontitud coordinativa de niños peruanos en función de la edad, sexo y estatus socioeconómico. Metodología: El tamaño de la muestra comprendió a 4,007 niños (femenino=1889; masculino $=$ 2118) entre los 6 y los 11 años de edad (niñas= 8,99; niños= 9,07) de escuelas del área metropolitana de Lima, Perú. La CoM fue determinado a través de la batería de pruebas KTK que comprende cuatro pruebas: equilibrio a la retaguardia (ER), saltos laterales (SL), saltos monopedales (SM) y transposición lateral (TL). El estatus socioeconómico (ESE) fue evaluado según la localización física de cada escuela (tipo de vecindad). Los análisis estadísticos exploratorios, descriptivos e inferenciales: análisis de varianza multivariable, función discriminante y chi cuadrado fueron realizados en SPSS 15. Las cartas percentílicas fueron elaboradas en el software LMS versión 1.32. Resultados: Existen incrementos significativos de valores medios de las pruebas de KTK en ambos sexos y a lo largo de la edad. Valores de reclasificación de los escolares en sus edades originales es bajo y presenta tendencia decreciente a lo largo de la edad $(30 \%$ a $23 \%$ y $30 \%$ a $20 \%$ en niñas y niños, respectivamente). La pertenencia a un determinado ESE no expresa la presencia de perfiles de desarrollo coordinativo diferenciados. Conclusiones. El desarrollo coordinativo es altamente específico a cada género. Se constata una tendencia generalizada en ambos sexos de expresar perfiles de desarrollo coordinativo inferiores a lo esperado para su edad. El ESE no es un predictor concluyente en el desarrollo de la coordinación motora de los niños.

\section{ABSTRACT}

Coordination readiness: multivariate profiles based upon age, sex, and socioeconomic status

Purpose: To describe motor coordination levels (CoM), their centile distribution, as well as identify the coordinative readiness of Peruvian children as a function of age, sex and socioeconomic status.

Methodology: Sample size included 4007 children ( $n=1889$ females; $n=2118$ males) between 6 and 11 years of age (young $=8,99$; children $=9,07)$ from several schools of the metropolitan area of Lima, Perú. CoM was assessed with the KTK, a battery of tests that includes: balancing backward (BB), hopping on one leg (HO), jumping sideways (JS) and shifting platforms (SP). Socioeconomic status (SES) was assessed according to the physical localization of each school (type of neighborhood). Exploratory, descriptive and inferential statistical analyses were used, including: multivariate analysis of variance, discriminant function and chi square. Percentile charts were constructed in the LMS software version 1.32. Results: Significant increases in mean values of CoM were found in both sexes, and across age groups. Reclassification values of $\mathrm{CoM}$ in their original ages are low and presents a decreased tendency throughout age (30\% to 23\% and $30 \%$ to $20 \%$ in girls and boys, respectively). SES is not a relevant predictor of differences in coordination profiles of children. Conclusions: The development of coordination is highly gender specific. There is a clear trend, in boys and girls, to show a coordination profile that is lower than expected for their chronological age. SES is not a conclusive predictor in the development of motor coordination profiles of children.

Key-words: coordination, readiness, reclassification, percentiles charts

Palabras-clave: prontitud coordinativa, reclasificación, cartas percentílicas 


\section{INTRODUCCIÓN}

La escuela es la institución más reconocida para el desarrollo y mejora de las facultades intelectuales, morales y físicas de niños y adolescentes, dado que los escolares representan el futuro de los pueblos y sus posibilidades de aporte a la sociedad, dependen de la calidad del proceso educativo. La adquisición y desarrollo de las habilidades de naturaleza ontogenética se hace a partir de una base de coordinación neuro-muscular progresiva, tomando en cuenta las cuatro fases: las respuestas reflejas, las habilidades fundamentales generales, las habilidades fundamentales específicas y las habilidades especializadas ${ }^{(1)}$. De acuerdo con Meinel y Schnabel (1976:5)(2), debemos comprender la complejidad de la tarea coordinativa como la armonización de todas las fuerzas internas $y$ externas, considerándose todos los grados de libertad del aparato de movimiento para una solución sensata de la tarea motora propuesta.

El desarrollo motor en los niños, enriquecido por las experiencias psicomotoras que acontecen en el día a día y por el carácter lúdico y variado de sus juegos, tiene que tomar en cuenta, necesariamente, la tarea disciplinadora, estructurada y orientada de la Educación Física, que incorpora una de las expresiones más ricas de la cultura humana - el Deporte(3). El respeto inalienable por las diferencias interindividuales que demuestran los niños que pertenecen a un determinado grado de escolaridad debe ser un principio presente en todo proceso pedagógico; por tanto, las estructuras didáctico-metodológicas, las estrategias de evaluación del proceso y del producto del proceso de enseñanza-aprendizaje deben tomar en cuenta estas diferencias. Asumir este presupuesto es reconocer que estamos frente a un problema inherente a las sesiones de Educación Física, en la que se manifiesta una respuesta diferenciada de los niños al aprendizaje motor de acuerdo con la prontitud coordinativa que presentan.

La preocupación por los beneficios que la escuela puede ofrecer a los niños en su proceso de formación, llevó a Brenner(4) a proponer por primera vez el concepto de prontitud al intentar hallar una respuesta adecuada a dos asuntos nucleares: (1) ¿Estará el niño listo para aprender a leer? (2) y ca escribir? Por otra parte, Bruner(5), en el contexto de los programas de enseñanza de la matemáticas y ciencias sociales para jóvenes, establece que uno de los principios a tener en cuenta en el proceso educativo es la de preocuparse por las experiencias y contextos que hacen al estudiante dispuesto y capaz de aprender (prontitud). En la literatura de las Ciencias del Deporte, el concepto fue introducido por Malina(6), Magill $(7,8)$ y Seefeldt $(9,10)$, siendo habitualmente definido de modo funcional acentuando la relación entre las capacidades de un individuo y las exigencias de una tarea o una actividad específica(11).

La prontitud coordinativa es en la actualidad una estructura conceptual compleja en pleno desarrollo, así como los procedimientos metodológicos y analíticos a ser utilizados para su determinación no han sido formulados expresamente, entre otras razones por presentar la coordinación motora una estructura multidimensional. Para los propósitos de nuestro estudio, la prontitud coordinativa será definida como una situación de equilibrio entre las exigencias particulares para la ejecución de actividades motoras cotidianas, así como para la adquisición, estabilización y diversificación de destrezas motoras gradualmente más complejas en diversos contextos de aprendizaje $\mathrm{y}$, las capacidades coordinativas actuales que posee el niño y el adolescente que le permita dar respuestas apropiadas a dichas exigencias. Por otro lado, la prontitud coordinativa definida operacionalmente está referida al posicionamiento de los individuos en una de las tres condiciones generadas a partir de los valores obtenidos en las pruebas del Test de Coordinación Corporal para Niños

(Körperkoordinationstest für Kinder, KTK) que fue desarrollado por Kiphard y Schilling ${ }^{(19)}$ y reclasificadas por medio del procedimiento de análisis de la función discriminante (FD). El niño o adolescente puede ser clasificado en el seno de su edad original, que refleja una prontitud coordinativa ajustada para su edad; en grupos con edades más avanzadas, que expresa un perfil coordinativo superior; o en grupos con edades más tempranas, que revela una insuficiencia de prontitud coordinativa.

La prontitud motora y la variabilidad de los niveles de aptitud física y del desarrollo coordinativo fueron abordadas en diferentes estudios, siendo los más referenciados los realizados en Portugal. Gomes(12), al estudiar a escolares de Matosinhos de ocho a diez años de edad y, al efectuar un análisis de Función 
Discriminante con los datos obtenidos de la batería KTK, encontró reclasificaciones correctas en niños del $44.0 \%$ y en niñas del $47.5 \%$. Por otro lado, con los valores obtenidos a partir de la batería AAHPERD las reclasificaciones en las edades originales fueron del $47.8 \%$ en los niños. Lopes y Maia ${ }^{(13)}$ hallaron en escolares açorianos de seis a los diez años un porcentaje reducido de reclasificación de los niños en sus grupos etarios naturales, siendo $33.1 \%$ y $33.5 \%$ en el sexo femenino y masculino, respectivamente. Sousa(14) halló, en niños de Amarante de los seis a los nueve años, que la tasa de reclasificación fue de $39.1 \%$ en niñas y $40.6 \%$ en niños. Lopes y Maia(15), al analizar los perfiles multivariados de la coordinación motora en escolares de seis a los diez años de edad pertenecientes a la Región Autónoma de los Açores, verificaron la existencia de perfiles de coordinación motora inferiores a los esperados para cada edad, siendo el porcentaje de reclasificados correctamente del $39.2 \%$ y $41.1 \%$ en niñas y niños, respectivamente. Fisberg(16) al estudiar a niños de dos a los seis años de edad pertenecientes a un estrato socio-económico bajo de la Prefectura de Sao Paulo en el Brasil, haciendo uso de la prueba de desarrollo de Denver, encontró que el $71 \%$ presentaba desempeño normal y en relación con el sexo no existían diferencias significativas, resultados que reflejan una prontitud adecuada para el aprendizaje, a pesar de pertenecer a contextos socioeconómicos desfavorecidos.

En países del hemisferio sur, específicamente en los de Sudamérica, al revisar la información disponible en la literatura sobre estudios relacionados con la prontitud coordinativa, no se encontró publicaciones que nos permitan tener una idea clara sobre el estado de los hechos, a pesar de que el número de niños $y$ adolescentes en edad escolar se incrementa sustantivamente año a año en estos pueblos.

En el Perú la situación se torna más preocupante por lo siguiente: La Ley General de Educación No 28044 tiene como objetivos principales la formación integral del educando en los aspectos físico, afectivo y cognitivo; el desarrollo de sus capacidades, valores y actitudes que permitan al educando a aprender a lo largo de toda su vida; así como el desarrollo de aprendizajes en los campos de las ciencias, las humanidades, la técnica, la cultura, el arte, la educación física y los deportes. Además, en el documento "Diseño Curricular Nacional de Educación Básica Regular Proceso de Articulación”(17), en relación con el Nivel Primario, la Educación Física como área curricular está considerada con dos horas semanales. A pesar de que en los documentos oficiales está explicitada su importancia, lo que acontece en realidad es que la inmensa mayoría de escolares de este nivel en todo el país no se beneficia de las sesiones de Educación Física, porque las autoridades de los sectores educativo y económico, al considerarlos no prioritarios, no deciden cubrir con profesores especialistas las plazas disponibles en esta área curricular.

Es de esperar en el campo del desarrollo motor cierta heterogeneidad interindividual, así como de acuerdo con posturas normativistas, es correcto pensar que, al interior de cada edad, los niños expresan un perfil coordinativo que las tornan homogéneas en cada edad(15). El estado de prontitud coordinativa es un asunto que despierta interés y preocupación en los padres y profesores, pues requieren conocer si durante el proceso educativo los perfiles coordinativos de los niños son los que se esperan para su edad o corresponden a niños con edades más avanzadas o con edades más tempranas. Es, en este contexto, que la importancia del estudio de la prontitud coordinativa y de la variabilidad interindividual del desarrollo coordinativo está orientada en dos aspectos: (a) en cuanto a su valor pedagógico como aspecto fundamental para el aprendizaje de una amplia gama de habilidades motoras, y (b) como elemento esencial que posibilitará la estructuración de programas didáctico-metodológicos apropiados respetando la variedad de los niveles de prontitud coordinativa de los niños. Por lo que la realización del presente estudio en intervalos etarios de seis a los once años de edad, así como en realidades socio-económicas diferenciadas, conllevó a que los objetivos del presente trabajo sean: (1) caracterizar el nivel de desarrollo coordinativo según género sexual a lo largo de la edad, (2) describir la distribución percentílica del comportamiento del desarrollo coordinativo según el género sexual a lo largo de la edad, y (3) identificar la prontitud coordinativa de los niños en función de la edad, el sexo y el estatus socioeconómico. 


\section{MATERIAL Y MÉTODOS Muestra}

La muestra fue constituida por 4007 niños de ambos sexos, con edades comprendidas entre los seis y los once años (Cuadro 1). Acudían a siete instituciones educativas, de las cuales cinco son de gestión estatal y dos de gestión privada; todas ubicadas en cuatro distritos del cono este de la ciudad de Lima (Lurigancho-Chosica, Chaclacayo, Ate-Vitarte y La Molina). Estas instituciones fueron seleccionadas intencionalmente de acuerdo con las facilidades otorgadas para realizar las observaciones, las cuales se realizaron entre los meses de setiembre de 2004 y julio de 2005. En todos los casos se solicitó la autorización de los padres o tutores y el consentimiento individual de cada niño, además del permiso otorgado por autoridades de las instituciones educativas participantes en el estudio.

Cuadro 1. Frecuencia absoluta y relativa de la muestra en función de la edad y sexo.

\begin{tabular}{ccccc}
\hline & \multicolumn{2}{c}{ Masculino } & \multicolumn{2}{c}{ Femenino } \\
\cline { 2 - 5 } Edad & $\mathbf{n}$ & $\mathbf{\%}$ & $\mathbf{n}$ & $\%$ \\
\hline 6 & 227 & 10,7 & 213 & 11,3 \\
7 & 296 & 14,0 & 270 & 14,3 \\
8 & 340 & 16,1 & 310 & 16,3 \\
9 & 372 & 17,5 & 321 & 17,0 \\
10 & 410 & 19,4 & 345 & 18,3 \\
11 & 473 & 22,3 & 430 & 22,8 \\
\hline Total: & $\mathbf{2 1 1 8}$ & & $\mathbf{1 8 8 9}$
\end{tabular}

Para la caracterización del estatus socio-económico (ESE) de los escolares, se utilizó como criterio la escuela donde cursaban estudios. Este criterio es válido sobre todo para ser utilizado en países en vías de desarrollo(18). Con un nivel socio-económico elevado (ESEE) fueron clasificados los estudiantes de escuelas privadas, con nivel socio-económico medio (ESEM) los de las escuelas estatales ubicadas en la zona urbana de cada distrito, y con nivel socio-económico bajo (ESEB) los pertenecientes a escuelas de zonas urbano-marginales.

\section{Evaluación de la coordinación motora}

La CoM fue evaluada a través del Test de

Coordinación Corporal para Niños (KTK) ${ }^{(19)}$, que

está constituida de cuatro pruebas: equilibrio en marcha hacia la retaguardia (ER), saltos laterales (SL), saltos monopedales (SM) y transposición lateral (TL). Los resultados de fiabilidad, estimados a partir del coeficiente de correlación intraclase $(\mathrm{R})$ en los equipos de evaluadores en el estudio piloto, fueron de 0.88 (ER), 0.90 (SL), 0.80 (SM) y 0.91 (TL).

\section{Procedimientos estadísticos}

Se realizó un análisis exploratorio de los datos con la finalidad de evaluar la normalidad de la distribución, identificar la presencia de "outliers", y obtener un cuadro descriptivo de las variables observadas. Las cartas percentílicas fueron elaboradas con la ayuda del modelo matemático-estadístico LMS presentado por Cole(20) y Cole \& Green(21), implementado en el software LMS versión 1.32 (A program for calculating age-related referente centiles). Para cuantificar la presencia de perfiles multivariados del desarrollo coordinativo en los grupos naturales de edad cronológica de los niños, se recurrió al procedimiento metodológico-analítico propuesto por Maia(14,15) que sugiere, en primer lugar, el análisis multivariado (MANOVA), seguida del análisis de la Función Discriminante. Para analizar las diferencias entre los estratos socioeconómicos se acudió al Chi Cuadrado. El nivel de significancia se mantuvo en $5 \%$. Los cálculos fueron realizados haciendo uso del programa estadístico SPSS 15.0.

\section{RESULTADOS}

En el Cuadro 2 se presentan las medidas descriptivas básicas de los resultados obtenidos por niños de ambos sexos en los diferentes intervalos etarios de las pruebas de la batería KTK. Se comprueba el incremento de los valores medios de cada prueba a lo largo de la edad, en ambos sexos. En las pruebas de ER y SM, los valores de las desviaciones estándar en torno a los valores medios reflejan la existencia de una variabilidad interindividual en ambos sexos. La magnitud de las desviaciones estándar es baja en la prueba de TL, que nos indica cierta homogeneidad de los resultados individuales en torno a los valores medios. En la prueba de SL las desviaciones estándar presentan un comportamiento diferente, siendo entre las edades de seis a los ocho años de edad más bajas en relación con el de edades más avanzadas; quiere decir, que en los tres primeros grupos existe mayor homogeneidad en los resultados en comparación con los grupos de nueve a once años de edad. 
Cuadro 2. Media y desviación estándar ( $M \pm d e$ ), valores mínimo (Mín.) y máximo [Máx.] de las cuatro pruebas de la batería KTK según edad y sexo.

\begin{tabular}{|c|c|c|c|c|c|c|c|c|c|c|c|c|c|}
\hline \multicolumn{2}{|c|}{ Pruebas KTK: } & \multicolumn{3}{|c|}{ Equilibrio a la Retaguardia } & \multicolumn{3}{|c|}{ Saltos Laterales } & \multicolumn{3}{|c|}{ Saltos Monopedales } & \multicolumn{3}{|c|}{ Transposición Lateral } \\
\hline Edad & Sexo & M \pm de & Mín. & Máx. & M \pm de & Mín. & Máx. & M \pm de & Mín. & Máx. & M \pm de & Mín. & Máx. \\
\hline \multirow[t]{2}{*}{6} & Mas. & $28,6 \pm 12,7$ & 4 & 61 & $24,9 \pm 6,6$ & 8 & 45 & $21,7 \pm 10,0$ & 2 & 51 & $13,1 \pm 2,9$ & 6 & 21 \\
\hline & Fem. & $32,4 \pm 13,1$ & 7 & 63 & $25,1 \pm 6,3$ & 13 & 53 & $17,4 \pm 7,9$ & 2 & 40 & $12,5 \pm 2,7$ & 7 & 21 \\
\hline \multirow[t]{2}{*}{7} & Mas. & $34,8 \pm 13,6$ & 3 & 69 & $29,5 \pm 7,2$ & 13 & 53 & $28,9 \pm 10,7$ & 2 & 57 & $15,3 \pm 3,1$ & 7 & 24 \\
\hline & Fem. & $37,0 \pm 13,5$ & 9 & 69 & $27,5 \pm 6,7$ & 9 & 46 & $22,4 \pm 8,9$ & 2 & 47 & $14,1 \pm 2,9$ & 8 & 25 \\
\hline \multirow[t]{2}{*}{8} & Mas. & $38,9 \pm 13,2$ & 9 & 72 & $33,4 \pm 8,8$ & 11 & 62 & $35,6 \pm 11,3$ & 6 & 68 & $17,2+2,9$ & 9 & 26 \\
\hline & Fem. & $39,4 \pm 12,9$ & 4 & 67 & $33,5 \pm 8,8$ & 12 & 58 & $28,8 \pm 8,9$ & 6 & 51 & $15,9 \pm 2,8$ & 8 & 25 \\
\hline \multirow[t]{2}{*}{9} & Mas. & $43,0 \pm 13,0$ & 13 & 72 & $40,1 \pm 10,0$ & 17 & 74 & $40,7 \pm 11,0$ & 11 & 69 & $18,6 \pm 3,0$ & 11 & 27 \\
\hline & Fem. & $43,9 \pm 13,4$ & 12 & 72 & $39,7 \pm 10,0$ & 18 & 63 & $34,3 \pm 10,2$ & 6 & 65 & $17,0 \pm 2,7$ & 10 & 25 \\
\hline \multirow[t]{2}{*}{10} & Mas. & $45,3 \pm 14,0$ & 13 & 72 & $42,7 \pm 11,2$ & 15 & 72 & $45,6 \pm 12,0$ & 16 & 72 & $19,6 \pm 3,6$ & 11 & 30 \\
\hline & Fem. & $47,0 \pm 12,9$ & 13 & 72 & $44,5 \pm 11,0$ & 16 & 74 & $38,9 \pm 10,9$ & 8 & 68 & $19,0 \pm 3,4$ & 10 & 31 \\
\hline \multirow[t]{2}{*}{11} & Mas. & $49,3 \pm 12,6$ & 13 & 72 & $49,9 \pm 10,8$ & 20 & 78 & $53,4 \pm 12,1$ & 21 & 72 & $19,6 \pm 3,6$ & 13 & 33 \\
\hline & Fem. & $49,2 \pm 12,2$ & 14 & 72 & $48,6 \pm 10,5$ & 23 & 80 & $45,2 \pm 11,6$ & 12 & 72 & $19,0 \pm 3,4$ & 12 & 28 \\
\hline
\end{tabular}

Los valores originales obtenidos por cada uno de los escolares en cada una de las cuatro pruebas de la batería KTK son extremadamente difíciles de ser interpretados en sí mismas. Es necesario recurrir a procedimientos de análisis que permitan obtener información de utilidad para los encargados de la formación de los niños, por lo que acudimos a hacer uso de los análisis referidos a la norma. En el Gráfico 1 se presentan las distribuciones percentílicas del comportamiento de los valores que corresponden a cada una de las cuatro pruebas del KTK según género sexual y a lo largo de la edad. Los valores percentílicos obtenidos por el método propuesto por Cole ${ }^{(20)}$ y Cole \& Green(21) para cada una de las pruebas del KTK según sexo e intervalos etarios son presentados en el Anexo 1. Desde el punto de análisis normativo, los percentiles $3,10,25,50,75,90$ y 97 son suficientes para describir la fuerte variabilidad que se encuentra en cada prueba. Los percentiles son medidas de posición relativa, que señalan, para un determinado valor, el porcentaje de individuos por encima y por debajo de ese valor; es decir, que al establecer las normas percentílicas y según su perfil de desarrollo coordinativo que manifiestan lo situamos al interior de su grupo, identificando de esta manera las diferencias interindividuales existentes.

Para el profesional de Educación Física y Deporte, así como para los profesores de aula y los padres encarga- dos de la educación de los niños conocer estos valores representa una información de enorme importancia, pues se constituye en valores de referencia, sobre todo para un sector de la población peruana, que posibilitará efectuar el seguimiento del desarrollo coordinativo. La utilización de las distribuciones y de los valores percentílicos estará orientado principalmente a la ubicación de cada escolar en el seno del grupo al que pertenece de acuerdo con el valor original obtenido. Por ejemplo, un niño de siete años de edad que en la prueba de TL obtiene 14 puntos. Consultando el Cuadro 4 del Anexo 1, constatamos que el resultado del niño se sitúa un punto por encima del percentil 25 , por lo que podemos indicar que el niño está en una posición de la distribución, con $25 \%$ de los niños debajo y $75 \%$ de los niños por encima de su resultado. Sin embargo, existe la posibilidad de establecer puntos de corte que permitan estipular que los valores por debajo del percentil 10 expresan un desempeño coordinativo relativamente insuficiente, los valores que se ubican entre el percentil 10 y 90 son considerados ajustados para una edad y sexo determinado, mientras que los valores por encima del percentil 90 son considerados superiores (22). De esta manera, procuramos establecer una ligazón coherente con la prontitud coordinativa, de forma que los profesionales de la Educación Física y Deporte tengan una herramienta de diagnóstico más accesible para su trabajo diario. 
Como ejemplo podemos señalar que una niña de seis años de edad que en la prueba de SL haya obtenido un resultado de 25 puntos estará ubicada por encima del percentil 25 y su desempeño coordinativo será lo
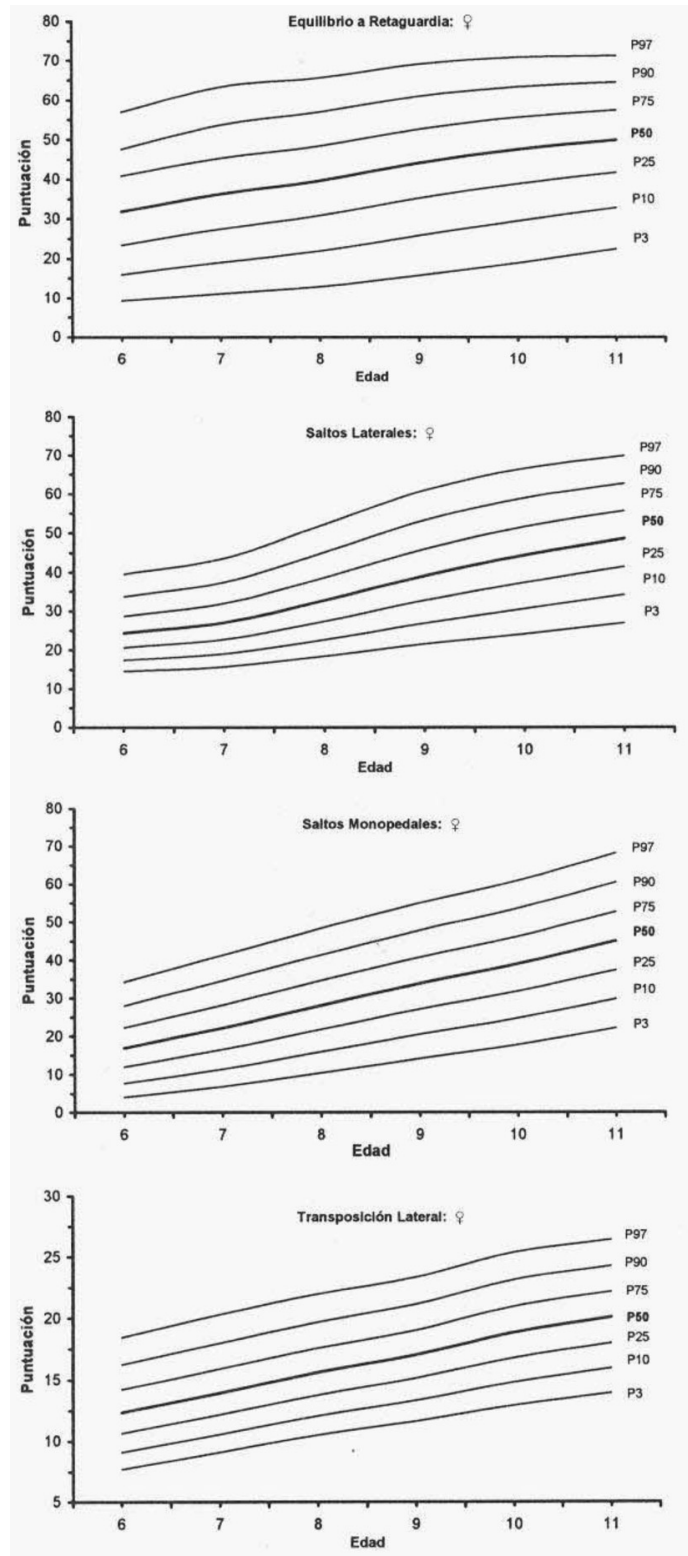

esperado para su edad; mientras que otra niña con una edad de diez años y con el mismo puntaje estará ubicada por debajo del percentil 3 y su desempeño coordinativo será considerada insuficiente.
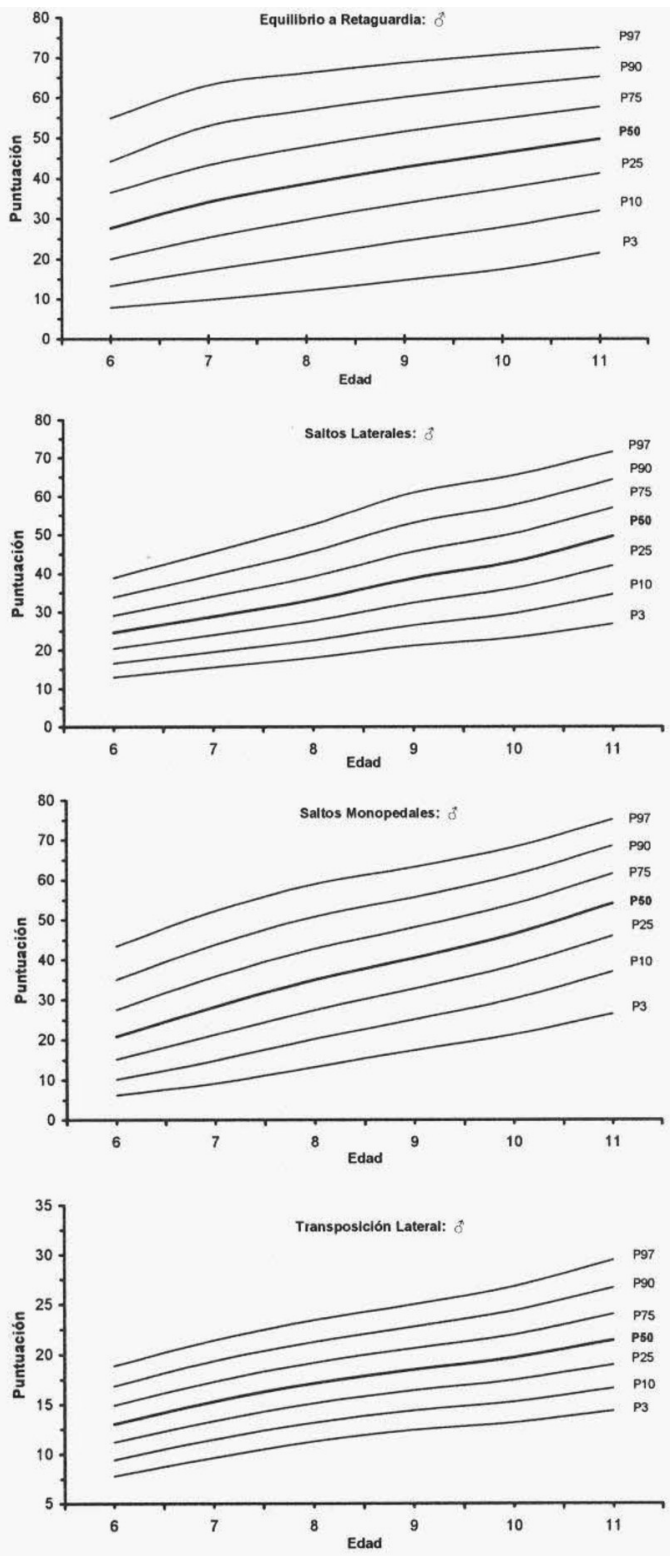

Gráfico 1. Distribución percentílica de las cuatro pruebas de la batería KTK de niñas y niños en función de la edad. 
Los resultados del análisis multivariado constatan diferencias significativas entre las medias de los diferentes componentes del desarrollo coordinativo a lo largo de la edad en el grupo de las niñas $(\Lambda$ de Wilks $=0.027, F(4: 1880)=16723.53, p=0.001)$ como en los escolares pertenecientes al sexo masculino $(\Lambda$ de Wilks=0.030, $\mathrm{F}(4: 2109)=17257.17, \mathrm{p}=0.001)$. Las matrices de reclasificación con frecuencias absolutas y en porcentajes se presentan en los Cuadros 3 y 4, respectivamente para las niñas y niños. Los valores que están en negrita corresponden a escolares cuya prontitud coordinativa es la esperada para su edad original; los otros, en cada línea, corresponden a escolares "mal clasificados" -con prontitud coordinativa más avanzada o más retrasada de lo que es esperado para su edad original.
$\mathrm{Al}$ observar los seis escalones etarios, los porcentajes de reclasificación de las niñas en sus grupos naturales, por ejemplo, en su edad cronológica, sobre la base de sus perfiles multidimensionales de coordinación motora, se verifica que a los seis años el porcentaje de niñas bien clasificadas es del $65.26 \%$. A partir de esta edad, los valores presentan una tendencia decreciente hasta los diez años: $30.0 \%$ a los 7 años, $29.68 \%$ a los 8 años, $28.97 \%$ a los 9 años, $23.19 \%$ a los 10 años, mientras que a los 11 años fue del $61.40 \%$. Se trata de valores de reclasificación muy reducidos que expresan las niñas en sus grupos originales de edad.

Por otro lado, los niños bien clasificados a los seis años es del 68.28\% (155 niños en 227). A partir de esta edad, los porcentajes son más bajos: $30.07 \%$ (89 en 296 niños) a los 7 años, 29.71\% (101 en 340

Cuadro 3. Cuadro de reclasificación de las niñas sobre la base de los resultados significativos de la función discriminante (FD) encontrada*. En negrita se encuentran los valores respecto a las niñas correctamente reclasificadas en sus edades (i.e. con un perfil coordinativo esperado para su edad).

\begin{tabular}{clcccccr}
\hline & \multicolumn{7}{c}{ Grupos naturales de edad (previsión sobre la base de los resultados de la FD) } \\
\cline { 2 - 8 } Edades & \multicolumn{1}{c}{$\mathbf{6}$} & $\mathbf{7}$ & $\mathbf{8}$ & $\mathbf{9}$ & $\mathbf{1 0}$ & $\mathbf{1 1}$ & TOTAL \\
\hline $\mathbf{6}$ & $\mathbf{1 3 9 ( 6 5 , 2 6 \% )}$ & $49(23,00 \%)$ & $19(8,92 \%)$ & $5(2,35 \%)$ & $1(0,47 \%)$ & $0(0,00 \%)$ & 213 \\
7 & $111(41,11 \%)$ & $\mathbf{8 1 ( 3 0 , 0 0 \% )}$ & $52(19,26 \%)$ & $17(6,30 \%)$ & $7(2,59 \%)$ & $2(0,74 \%)$ & 270 \\
$\mathbf{8}$ & $38(12,26 \%)$ & $75(24,19 \%)$ & $\mathbf{9 2 ( 2 9 , 6 8 \% )}$ & $66(21,29 \%)$ & $30(9,68 \%)$ & $9(2,90 \%)$ & 310 \\
9 & $20(6,23 \%)$ & $32(9,97 \%)$ & $65(20,25 \%)$ & $\mathbf{9 3 ( 2 8 , 9 7 \% )}$ & $58(18,07 \%)$ & $53(16,51 \%)$ & 321 \\
10 & $6(1,74 \%)$ & $12(3,48 \%)$ & $46(13,33 \%)$ & $64(18,55 \%)$ & $\mathbf{8 0 ( 2 3 , 1 9 \% )}$ & $137(39,71 \%)$ & 345 \\
11 & $4(0,93 \%)$ & $2(0,47 \%)$ & $26(6,05 \%)$ & $50(11,63 \%)$ & $84(19,53 \%)$ & $\mathbf{2 6 4}(\mathbf{6 1 , 4 0 \% )}$ & 430 \\
\hline
\end{tabular}

* $\left(\Lambda\right.$ de Wilks=0,412, $\left.\quad \chi^{2}=1668,447, p<0.001\right)$

$\%$ Total de reclasificación correcta $=39,7 \%$

Cuadro 4. Cuadro de reclasificación de las niños sobre la base de los resultados significativos de la función discriminante (FD) encontrada*. En negrita se encuentra los valores respecto a los niños correctamente reclasificados en sus edades (i.e. con un perfil coordinativo esperado para su edad).

\begin{tabular}{cccccccr}
\hline & \multicolumn{7}{c}{ Grupos naturales de edad (previsión sobre la base de los resultados de la FD) } \\
\cline { 2 - 8 } Edades & $\mathbf{6}$ & $\mathbf{7}$ & $\mathbf{8}$ & $\mathbf{9}$ & $\mathbf{1 0}$ & $\mathbf{1 1}$ & TOTAL \\
\hline $\mathbf{6}$ & $\mathbf{1 5 5 ( 6 8 , 2 8 \% )}$ & $46(20,26 \%)$ & $21(9,25 \%)$ & $5(2,20 \%)$ & $0(0,00 \%)$ & $0(0,00 \%)$ & 227 \\
$\mathbf{7}$ & $100(33,78 \%)$ & $\mathbf{8 9 ( 3 0 , 0 7 \% )}$ & $73(24,66 \%)$ & $25(8,45 \%)$ & $8(2,70 \%)$ & $1(0,34 \%)$ & 296 \\
$\mathbf{8}$ & $35(10,29 \%)$ & $85(25,00 \%)$ & $\mathbf{1 0 1 ( 2 9 , 7 1 \% )}$ & $60(17,65 \%)$ & $41(12,06 \%)$ & $18(5,29 \%)$ & 340 \\
$\mathbf{9}$ & $16(4,30 \%)$ & $28(7,537 \%)$ & $104(27,96 \%)$ & $\mathbf{8 9 ( 2 3 , 9 2 \% )}$ & $75(20,16 \%)$ & $60(16,13 \%)$ & 372 \\
$\mathbf{1 0}$ & $11(2,68 \%)$ & $26(6,34 \%)$ & $82(20,00 \%)$ & $63(15,37 \%)$ & $\mathbf{8 4 ( 2 0 , 4 9 \% )}$ & $144(35,12 \%)$ & 410 \\
11 & $3(0,63 \%)$ & $9(1,90 \%)$ & $25(5,29 \%)$ & $46(9,73 \%)$ & $67(14,16 \%)$ & $\mathbf{3 2 3}(68,29 \%)$ & 473 \\
\hline
\end{tabular}


niños) a los 8 años, $23.92 \%$ (89 en 372 niños) a los 9 años, $20.49 \%$ (84 en 410 ) a los 10 años y $68.29 \%$ (323 en 473 niños) a los 11 años. Similar a los valores de las niñas, los porcentajes de niños reclasificados en sus grupos originales de edad son reducidos. Es evidente la presencia en porcentajes altos de la "mala reclasificación". Por un lado existen niños en ambos sexos que en su edad expresan un perfil de prontitud coordinativa que es característica de sus colegas de edades más avanzadas, como es, por ejemplo, el caso de los seis años, en que existe 49 niñas y 46 niños cuyos perfiles multidimensionales de desarrollo coordinativo corresponden a la edad de siete años y así por delante. Del mismo modo a los siete, ocho, nueve y diez años de edad existen niños de ambos sexos con desarrollo coordinativo avanzado para su edad. Pero, lo que denota mayor preocupación es la presencia de escolares cuyo perfil corresponde a los de sus colegas de edad más baja. Por ejemplo, a los siete años hay 111 niñas y 100 niños que poseen un perfil de desarrollo coordinativo de colegas de seis años de edad. A los diez años
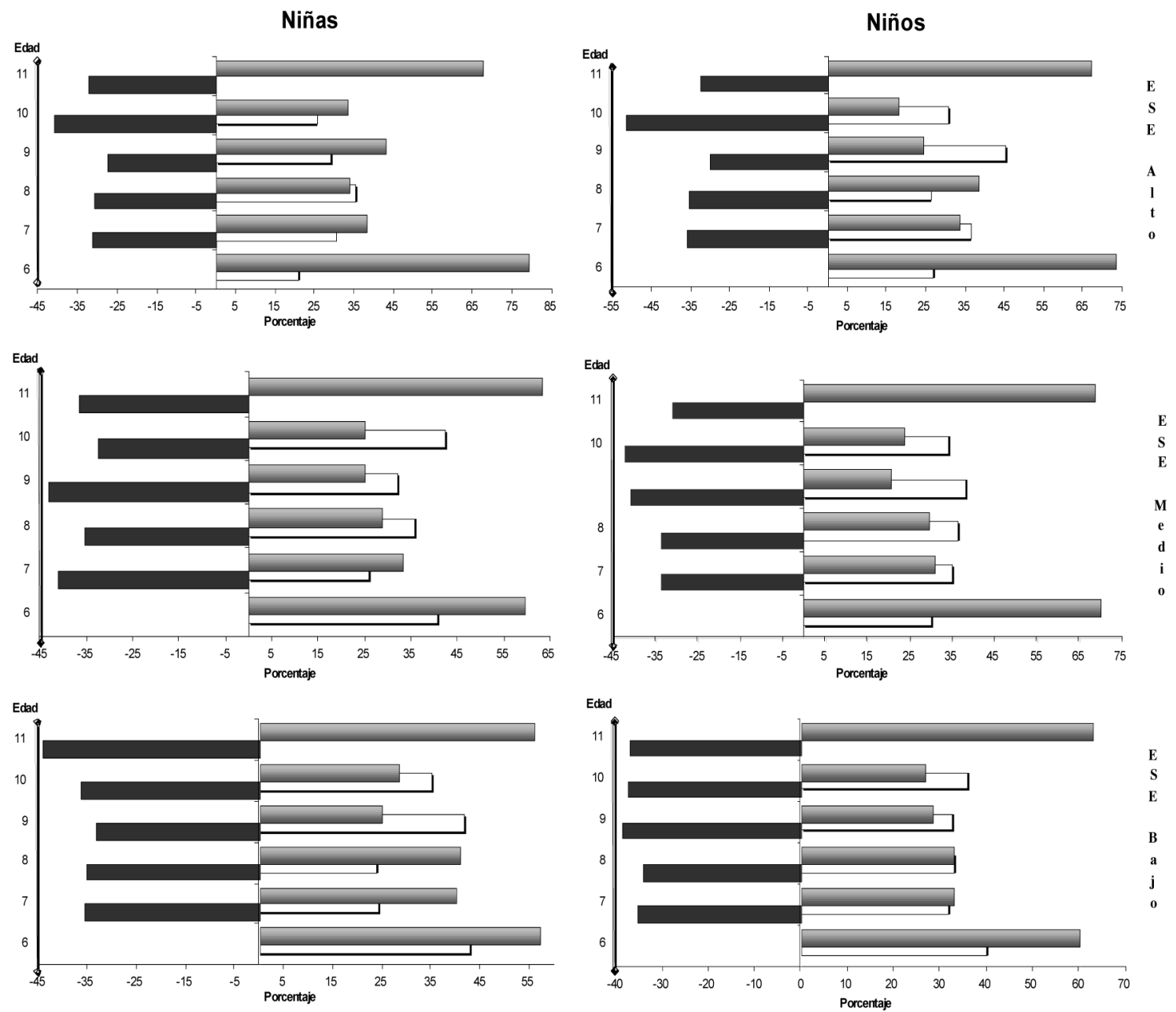

EDADATRASADA $\square$ EDADAVANADA $\square$ EDADDEORIGAN

Gráfico 2. Gráficos de reclasificación de niñas y niños basados en los resultados significativos de la función discriminante (FD) encontrada. Porcentaje de escolares reclasificados en su edad original, en edades anteriores y en edades avanzadas de acuerdo con el ESE. 
hay un $37.1 \%$ (128 en 345 niñas) y $44.39 \%$ (182 en 410 niños) cuyos perfiles multidimensionales los ubican en edades más bajas.

La pertenencia a un determinado estatus socioeconómico será un aspecto que pueda explicar la presencia importante de escolares con perfiles de desarrollo coordinativo que correspondan a edades más bajas. Para esclarecer esta preocupación se presenta gráficamente el comportamiento de los escolares reclasificados correctamente en sus grupos originales de edad, así como el porcentaje de niños clasificados en grupos de edad más avanzada y en grupos de edad más baja de acuerdo con intervalos etarios, género sexual y ESE (Gráfico 2). Al establecer comparaciones entre los tres grupos socioeconómicos en cada intervalo etario y por género sexual, los resultados del $\chi^{2}$ permiten verificar la existencia de diferencias significativas sólo en las niñas de nueve años de edad $\left(\chi^{2}=12.239, p=0.016\right)$. Este grupo contiene a 372 niñas, de las cuales un $29.0 \%$ pertenecen a los reclasificados en su edad de origen, un $34.3 \%$ ubicadas en edades avanzadas y un $36.8 \%$ a grupos de edad mas baja.

En el resto de las fajas etarias del grupo femenino y masculino no se constataron diferencias estadísticamente significativas. Estos resultados indican de manera inequívoca que la pertenencia a un determinado estrato socioeconómico no es un factor importante para la presencia de perfiles de desarrollo coordinativo diferenciados. En cada uno de los estratos socioeconómicos las proporciones de escolares que pertenecen a uno de los tres tipos de reclasificaciones no conllevan a la presencia de diferencias significativas. La atención por parte de los profesores y padres de familia debe darse de manera indistinta al interior de cada escuela y familia, sin tomar en cuenta el tipo de gestión educativa o estrato socioeconómico al que corresponden.

\section{DISCUSIÓN}

Una de las constataciones que surge del presente estudio es que, en las pruebas de SL y TL, los niños presentan valores medios superiores en todos los intervalos etarios en relación con las niñas, el mismo comportamiento se verifica en la prueba de SL a excepción del grupo etario de los diez años. Por otro lado, en la prueba de ER son las niñas quienes presentan valores medios elevados en la mayoría de las fajas etarias. Estos resultados corroboran los valores referidos en estudios con niños en edad escolar realizados en Portugal (23,24). Por otro lado, el comportamiento de los valores medios en la prueba de ER que favorece a las niñas son similares a las encontradas en los estudios de Kiphard e Schilling ${ }^{(19)}$ en los primeros cinco escalones etarios; a los de Andrade(24), a los ocho y nueve años de edad, mientras que sólo a los seis años tienen comportamientos idénticos a lo reportado por Maia y Lopes(23).

Las diferencias entre las medias de los diferentes componentes del desarrollo coordinativo a lo largo de la edad resultaron significativas en el grupo de niñas y niños. Estos resultados permiten subrayar la necesidad de considerar en estudios futuros los efectos del proceso de crecimiento y maduración en el que están inmersos los niños en estos escalones etarios, así como la importancia que poseen las experiencias motoras orientadas pedagógicamente en las sesiones de Educación Física en las escuelas y las espontáneas que acontecen fuera de ellas. Esta información corrobora lo señalado por Willimczik ${ }^{(25)}$ en relación con la existencia de un padrón de desarrollo específico de cada sexo.

Las cartas de distancia cumplen una función primordial en el seguimiento y monitorización del desarrollo coordinativo de los niños en edad escolar. Un instrumento válido no sólo para los profesores de Educación Física, sino también para los padres de familia, así como para los profesionales de la salud involucrados en la vigilancia del desarrollo coordinativo. A pesar de que la muestra del presente estudio es importante, no representa a la población escolar peruana en su integridad, por lo que su utilización debe ser referencial.

A partir de los valores que alcanzó cada uno de los niños en las cuatro pruebas de la batería KTK es posible definir un perfil multidimensional de la configuración de su desarrollo coordinativo(26). Los resultados que se presentan en las matrices de reclasificación permiten verificar el número de niños cuyo perfil de desarrollo coordinativo está de acuerdo con su edad actual o corresponde a edades más avanzadas o más atrasadas. Sin embargo, para el análisis de los resultados consideramos los escalones 
etarios entre los siete y los diez años de edad, dado que el grupo de seis años no tiene grupos de edad que los antecedan y los de once tampoco presentan grupos con edades más avanzadas, lo que no permite una reclasificación adecuada en estos dos grupos que se ubican en los extremos.

En los valores de reclasificación que corresponden a las niñas, verificamos que entre los siete y diez años de edad el porcentaje de escolares clasificadas en sus edades originales es muy bajo y presenta una tendencia claramente descendente (30\% a $23 \%)$. Estos resultados son similares a los encontrados en escolares portugueses, donde los resultados disminuyen del 28 al $24 \%$ entre los siete y los nueve años de edad. Un comportamiento similar se observa en el grupo de los niños (30\% a 20\%), notándose la misma tendencia en los niños portugueses cuyos porcentajes decrecen del 30 al 21\%(26). Sin duda, estos resultados son de mucha preocupación, cuando comprobamos que el perfil de desarrollo coordinativo de sólo un $33 \%$ de niñas y un $30 \%$ de niños corresponde a su edad original, a pesar de que existe un 22 y $24 \%$ de niñas y niños cuyos perfiles corresponden a edades más avanzadas. Sin embargo, el porcentaje elevado de niñas y niños reclasificados en edades que anteceden a la edad original $(44 \%$ y $46 \%$, respectivamente) explicita la existencia de un problema serio que expresa una insuficiencia de prontitud coordinativa.

Los resultados hallados parecen verificar que las deficiencias que se observan en el desarrollo coordinativo no presentan una tendencia definida hacia un determinado sexo, pues la magnitud de los valores porcentuales entre cada grupo no es muy amplia en las tres categorías de prontitud coordinativa. Este hecho es explicado por las constataciones que señalan que las diferencias de los sexos en la expresión de las capacidades coordinativas se hacen más notorias a partir de los trece años (27).

La pertenencia de los escolares a un determinado estatus socioeconómico no expresa la presencia de perfiles de desarrollo coordinativo diferenciados. Tomando en cuenta los escalones etarios de los siete a los diez años de edad, se verificó en general que el ESE no ejerce una influencia directa en las respuestas diferenciadas de los escolares al aprendizaje motor y a la posibilidad de presentar perfiles más elevados de prontitud coordinativa o a expresar insuficiencias de prontitud coordinativa. Las diferencias estadísticas encontradas en las niñas pertenecientes al intervalo etario de los nueve años, responde entre otros factores a los niveles diferenciados de crecimiento que empiezan a presentar las niñas en esta edad dependiendo del estrato al que pertenecen. Estos resultados exigen a los profesores de Educación Física que trabajan en instituciones educativas estatales o privadas, ubicadas en zonas urbanas, urbano-marginales o rurales, a realizar evaluaciones periódicas del desarrollo coordinativo que les permita obtener información actualizada de sus alumnos. Dos problemas importantes surgen a partir de los resultados analizados, uno relacionado con los escolares cuyos perfiles corresponden a escolares de edades más avanzadas y la otra con aquellos que presentan perfiles que pertenecen a grupos etarios más bajos. Con relación a la primera situación, es preciso que los contenidos de las sesiones de clase de Educación Física se redimensionen hacia los valores más elevados de prontitud coordinativa que presentan los escolares (26). En el segundo caso, la situación se torna más preocupante, porque los escolares que pertenecen a este grupo que reflejan insuficiencia de prontitud coordinativa, requieren que los contenidos de las clases de Educación Física sean adecuados a las necesidades de estos escolares, de manera tal que puedan alcanzar niveles de desarrollo de las capacidades coordinativas propias para su edad sobre la base de la cantidad y calidad de experiencias motoras. Un aspecto que no se debe dejar de lado se refiere a que esta insuficiencia de prontitud coordinativa puede repercutir negativamente en el logro de las capacidades cognoscitivas, específicamente en el desarrollo de las habilidades viso-espaciales. Estudios han expuesto que niños que presentaban dificultades en las pruebas cognoscitivas no demostraban deficiencias en las habilidades motoras; en cambio, la mayoría de niños que demostraban deficiencias motoras además presentaban dificultades en las pruebas cognoscitivas (28,29).

\section{CONCLUSIONES}

En ambos sexos y a lo largo de la edad en todas las pruebas de la batería KTK, existe un incremento significativo de los valores medios, que refleja la pre- 
sencia de un desarrollo coordinativo específico para cada sexo, por ende la plasticidad del desarrollo de la coordinación motora.

Se verifica en ambos sexos la presencia de perfiles de coordinación motora inferiores a aquellos que son esperados para sus edades originales. Esta situación traduce una fuerte insuficiencia en aspectos del desarrollo coordinativo en los distintos escalones etarios. El pertenecer a un determinado estatus socioeconómico no es un factor concluyente para definir un determinado perfil de prontitud coordinativo en los diferentes grupos de edad estudiados.

Los resultados encontrados reafirman la necesidad imperiosa de reestructurar los programas de Educación Física en el Nivel Primario y que éstos sean asumidos por profesionales debidamente formados que puedan producir efectos beneficiosos en el desarrollo coordinativo y garanticen un significativo incremento de las capacidades motoras y deportivas en los escolares peruanos. Este hecho conducirá a un perfeccionamiento progresivo de las estructuras que aseguren el desarrollo motor en niños y adolescentes que les permita ejecutar correcta y adecuadamente las acciones motoras en el hogar, la escuela y en los lugares públicos donde interactúan con sus pares.

Por último, se hace necesario incluir en futuros estudios sobre el desarrollo coordinativo en niños y adolescentes la variable maduración así como otros factores que pueden afectar positiva o negativamente la prontitud coordinativa.

\section{AGRADECIMIENTOS}

Los autores agradecen a la Fundación para la Ciencia y la Tecnología de Portugal por la beca de manutención SFRH/BM/15929/. Extendemos nuestro agradecimiento a los estudiantes de Educación Física de la UNE que participaron en la recolección de datos.

\section{CORRESPONDÊNCIA}

\section{Alcibíades Bustamante Valdivia}

Universidade do Porto, Faculdade de Desporto

Laboratório de Cineantropometria e Estatística

Aplicada

Rua Plácido Costa 91,

4200-450 Porto, Portugal

Fax: + 351225.500.689

E-mail: huanta2609@yahoo.es 


\section{BIBLIOGRAFIA}

1. Gallahue D (1982). Understanding motor development in children. New York: Wiley.

2. Meinel K (1987). Motricidade I: Teoria da motricidade esportiva sob o aspecto pedagógico. Ao Livro Técnico S/A, col. Educação Física, Rio de Janeiro.

3. Maia JAR, Lopes VP (2002). Estudo do crescimento somatico, aptidão física, actividade física e capacidade de coordenação corporal de crianças do $1^{\circ}$ Ciclo do Ensino Básico da Região Autónoma de Açores. Porto: FADEUP, Portugal.

4. Brenner A (1957). Nature and meaning of readiness for school. Merrill-Palmer Quarterly, 3: 114-135.

5. Bruner J (1973). Going Beyond the Information Given. New York: Norton.

6. Malina R.M (1986). Readiness for competitive sport. In: M. R. Weiss; D. Gould (eds.). Sport for children and Youths. Human Kinetics. Champaign.

7. Magill V (1982). Critical periods as optimal readiness for learning sport skills. In FL Smoll, RA Magill e MG Ash (eds.). Children in sport, $3^{\mathrm{a}}$ ed. Champaign: Human Kinetics.

8. Magill V (1988). Critical periods as optimal readiness for learning sport skills. In FL Smoll, RA Magill e MG Ash (eds.). Children in sport, $3^{\text {a }}$ ed. Champaign: Human Kinetics.

9. Seefeldt V (1982). The concept of readiness applied to motor skill acquisition. In FL Smoll, RA Magill e MG Ash (eds.). Children in sport, $3^{\text {a }}$ ed. Champaign: Human Kinetics.

10. Seefeldt V (1988). The concept of readiness applied to motor skill acquisition. In FL Smoll, RA Magill e MG Ash (eds.). Children in sport, $3^{\text {a }}$ ed. Champaign: Human Kinetics.

11. Malina R.M (1993). Youth sports: readiness, selection and trainability. In W Duquet, JAP Day (eds.). Kinathropometry $I V$. Londres: E\&FN Spon.

12. Gomes M.P.B.B (1996). Coordenação, aptidão física e variáveis do envolvimento. Estudo em crianças do $1^{\circ}$ ciclo de ensino de duas freguesias do concelho de Matosinhos. Tese de doutoramento. FCDEF, Porto: Universidade do Porto.

13. Lopes VP, Maia JAR (2004). Aptidão física associada á saúde da população escolar (6 a 10 anos de idade) do Arquipélago dos Açores, Portugal. Rev Brás Cioneantropom Desempenho Hum, 6 (2): 7-16.

14. Sousa MC, Maia J (2005). Crescimento somático, Actividade física e Aptidão física Associada á Saúde. Um estudo populacional nas crianças do $1^{\circ}$ Ciclo do Ensino Básico do Concelho de Amarante. FCDEF - Câmara Municipal de Amarante.

15. Lopes VP, Maia JAR (2003). Estudo do nível de desenvolvimento da coordenação motora da população escolar (6 a 10 anos de idade) da Região Autónoma dos Açores. Revista Portuguesa de Ciências do Desporto, 3 (1): 47-60

16. Fisberg M, et al (1997). Comparação do desempenho de pré-escolares, mediante teste de desenvolvimento de Denver, antes e após intervenção nutricional. Rev Ass Med Brasil, 43(2): 99-104

17. Ministerio de Educación (2005). Diseño Curricular Nacional de Educación Básica Regular. Lima, Perú.

18. Prista A, Marques A, Maia J (1997). Relationship Between Physical Activity, Socioeconomic Status, and Physical Fitness of 8-15-Year-Old Youth From Mozambique. American Journal of Human Biology, 9: 449-457.
19. Kiphard BJ, Schilling F (1974). Körperkoordinations Test für Kinder. Beltz Test GmbH, Weinheim.

20. Cole TJ (1990). The LMS method for constructing normalized growth standards. Eur J Clin Nutr, 44(1):45-60.

21. Cole TJ, Green PJ (1992). Smoothing reference centile curves: the LMS method and penalized likelihood. Stat Med., 11(10): 1305-1319.

22. Maia JA, Lopes VP, Bustamante A, Garganta R, Seabra A, Fonseca A, Freitas D, Prista A, Cardoso M. (2007). Crescimento e desempenho motor de crianças e jovens Açorianos. Cartas de referencia para uso em Educação Física, Desporto, Pediatria e Nutrição. Terceira e Porto: Direcção Regional do Desporto da Região Autónoma dos Açores e Faculdade do Desporto da Universidade do Porto.

23. Maia JA, Lopes VP (2007). Crescimento e desenvolvimento de crianças e jovens Açorianos. O que pais, professores, pediatras e nutricionistas gostariam de saber. Terceira e Porto: Direcção Regional do Desporto da Região Autónoma dos Açores e Faculdade do Desporto da Universidade do Porto.

24. Andrade MJLA (1996). Coordenação motora. Estudo em crianças do ensino básico na Região Autónoma da Madeira. [Dissertação de mestrado]. Porto: FCDEF, Universidade de Porto.

25. Willimczik K (1980). Development of motor control capability (body coordination) of 6-to 10-year-old children: Results of a Longitudinal Study. In M Ostyn, G Beunen, J Simons (eds.). Kinanthropometry II. Baltimore: University Park Press, 328-346.

26. Lopes V, Maia JA, Silva R, Seabra A, Morais F (2003). Estudo do nível de desenvolvimento da coordenação motora da população escolar (6 a 10 anos de idade) da Região Autónoma dos Açores. Revista Portuguesa de Ciências do Desporto, 3 (1): 47-60.

27. Hirtz P, Schielk E. (1986). O desenvolvimento das capacidades coordinativas nas crianças, nos adolescentes e nos jovens adultos. Horizonte, III serie, 15: 83-88.

28. Perera H. (2005). Readiness for school entry: a community survey. Public Health, 119: 283-289.

29. Wilson PH, McKenzie BE. (1998). Information processing deficits associated with developmental coordination disorder: a meta-analysis of research findings. J Child Psychol Psychiatry, 39(6):829-40 


\section{ANEXOS}

1. Valores percentílicos de la prueba de Equilibrio a Retaguardia de la batería KTK de niñas y niños.

\begin{tabular}{c|ccccccc}
\multicolumn{7}{c}{ Equilibrio a Retaguardia: niñas } \\
\hline Edad & $\mathbf{P}_{\mathbf{3}}$ & $\mathbf{P}_{\mathbf{1 0}}$ & $\mathbf{P}_{\mathbf{2 5}}$ & $\mathbf{P}_{\mathbf{5 0}}$ & $\mathbf{P}_{\mathbf{7 5}}$ & $\mathbf{P}_{\mathbf{9 0}}$ & $\mathbf{P}_{\mathbf{9 7}}$ \\
\hline $\mathbf{6}$ & 9 & 16 & 23 & 32 & 41 & 48 & 57 \\
$\mathbf{7}$ & 11 & 19 & 27 & 36 & 45 & 54 & 63 \\
$\mathbf{8}$ & 13 & 22 & 31 & 40 & 48 & 57 & 66 \\
$\mathbf{9}$ & 16 & 26 & 35 & 44 & 53 & 61 & 69 \\
10 & 19 & 29 & 39 & 48 & 56 & 63 & 71 \\
$\mathbf{1 1}$ & 22 & 33 & 42 & 50 & 58 & 65 & 71 \\
\hline
\end{tabular}

\begin{tabular}{c|ccccccc}
\multicolumn{7}{|c}{ Equilibrio a Retaguardia: niños } \\
\hline Edad & $\mathbf{P}_{\mathbf{3}}$ & $\mathbf{P}_{\mathbf{1 0}}$ & $\mathbf{P}_{\mathbf{2 5}}$ & $\mathbf{P}_{\mathbf{5 0}}$ & $\mathbf{P}_{\mathbf{7 5}}$ & $\mathbf{P}_{\mathbf{9 0}}$ & $\mathbf{P}_{\mathbf{9 7}}$ \\
\hline $\mathbf{6}$ & 8 & 13 & 20 & 28 & 36 & 44 & 55 \\
$\mathbf{7}$ & 10 & 17 & 25 & 34 & 43 & 53 & 63 \\
$\mathbf{8}$ & 12 & 21 & 30 & 39 & 48 & 57 & 66 \\
$\mathbf{9}$ & 15 & 24 & 34 & 43 & 52 & 60 & 69 \\
10 & 17 & 28 & 37 & 46 & 55 & 63 & 71 \\
11 & 21 & 32 & 41 & 50 & 58 & 65 & 73 \\
\hline
\end{tabular}

2. Valores percentílicos de la prueba de Saltos Laterales de la batería KTK de niñas y niños.

\begin{tabular}{c|ccccccc}
\multicolumn{7}{c}{ Saltos Laterales: niñas } \\
\hline Edad & $\mathbf{P}_{\mathbf{3}}$ & $\mathbf{P}_{\mathbf{1 0}}$ & $\mathbf{P}_{\mathbf{2 5}}$ & $\mathbf{P}_{\mathbf{5 0}}$ & $\mathbf{P}_{\mathbf{7 5}}$ & $\mathbf{P}_{\mathbf{9 0}}$ & $\mathbf{P}_{\mathbf{9 7}}$ \\
\hline $\mathbf{6}$ & 15 & 17 & 21 & 24 & 29 & 34 & 40 \\
$\mathbf{7}$ & 16 & 19 & 23 & 27 & 32 & 37 & 44 \\
$\mathbf{8}$ & 18 & 23 & 27 & 33 & 39 & 45 & 52 \\
$\mathbf{9}$ & 21 & 27 & 33 & 39 & 46 & 53 & 61 \\
10 & 24 & 31 & 37 & 44 & 52 & 59 & 67 \\
11 & 27 & 34 & 41 & 49 & 56 & 63 & 70 \\
\hline
\end{tabular}

\begin{tabular}{c|ccccccc}
\multicolumn{7}{|c}{ Saltos Laterales: niños } \\
\hline Edad & $\mathbf{P}_{\mathbf{3}}$ & $\mathbf{P}_{\mathbf{1 0}}$ & $\mathbf{P}_{\mathbf{2 5}}$ & $\mathbf{P}_{\mathbf{5 0}}$ & $\mathbf{P}_{\mathbf{7 5}}$ & $\mathbf{P}_{\mathbf{9 0}}$ & $\mathbf{P}_{\mathbf{9 7}}$ \\
\hline $\mathbf{6}$ & 13 & 17 & 20 & 25 & 29 & 34 & 39 \\
$\mathbf{7}$ & 16 & 20 & 24 & 29 & 34 & 40 & 46 \\
8 & 18 & 23 & 28 & 33 & 39 & 46 & 53 \\
$\mathbf{9}$ & 21 & 27 & 32 & 39 & 46 & 53 & 61 \\
10 & 23 & 30 & 36 & 43 & 50 & 58 & 66 \\
11 & 27 & 35 & 42 & 50 & 57 & 64 & 72 \\
\hline
\end{tabular}

3. Valores percentílicos de la prueba de Saltos Monopedales de la batería KTK de niñas y niños.

\begin{tabular}{c|ccccccc}
\multicolumn{7}{c}{ Saltos Monopodales: niñas } \\
\hline Edad & $\mathbf{P}_{\mathbf{3}}$ & $\mathbf{P}_{\mathbf{1 0}}$ & $\mathbf{P}_{\mathbf{2 5}}$ & $\mathbf{P}_{\mathbf{5 0}}$ & $\mathbf{P}_{\mathbf{7 5}}$ & $\mathbf{P}_{\mathbf{9 0}}$ & $\mathbf{P}_{\mathbf{9 7}}$ \\
\hline $\mathbf{6}$ & 4 & 8 & 12 & 17 & 22 & 28 & 34 \\
$\mathbf{7}$ & 7 & 11 & 17 & 22 & 28 & 35 & 41 \\
$\mathbf{8}$ & 10 & 16 & 22 & 28 & 35 & 41 & 48 \\
$\mathbf{9}$ & 14 & 21 & 27 & 34 & 41 & 48 & 55 \\
10 & 18 & 25 & 32 & 39 & 46 & 54 & 61 \\
11 & 22 & 30 & 37 & 45 & 53 & 61 & 68 \\
\hline
\end{tabular}

\begin{tabular}{c|ccccccc}
\multicolumn{7}{|c}{ Saltos Monopodales: niños } \\
\hline Edad & $\mathbf{P}_{\mathbf{3}}$ & $\mathbf{P}_{\mathbf{1 0}}$ & $\mathbf{P}_{\mathbf{2 5}}$ & $\mathbf{P}_{\mathbf{5 0}}$ & $\mathbf{P}_{\mathbf{7 5}}$ & $\mathbf{P}_{\mathbf{9 0}}$ & $\mathbf{P}_{\mathbf{9 7}}$ \\
\hline $\mathbf{6}$ & 6 & 10 & 15 & 21 & 28 & 35 & 43 \\
$\mathbf{7}$ & 9 & 15 & 21 & 28 & 36 & 44 & 52 \\
$\mathbf{8}$ & 13 & 20 & 28 & 35 & 43 & 51 & 59 \\
9 & 17 & 25 & 33 & 41 & 48 & 56 & 63 \\
10 & 21 & 30 & 39 & 46 & 54 & 61 & 68 \\
11 & 27 & 37 & 46 & 54 & 62 & 69 & 75 \\
\hline
\end{tabular}

4. Valores percentílicos de la prueba de Transposición Lateral de la batería KTK de niñas y niños.

\begin{tabular}{c|ccccccc}
\multicolumn{7}{|c}{ Transposición Lateral: niñas } \\
\hline Edad & $\mathbf{P}_{\mathbf{3}}$ & $\mathbf{P}_{\mathbf{1 0}}$ & $\mathbf{P}_{\mathbf{2 5}}$ & $\mathbf{P}_{\mathbf{5 0}}$ & $\mathbf{P}_{\mathbf{7 5}}$ & $\mathbf{P}_{\mathbf{9 0}}$ & $\mathbf{P}_{\mathbf{9 7}}$ \\
\hline $\mathbf{6}$ & 8 & 9 & 11 & 12 & 14 & 16 & 18 \\
$\mathbf{7}$ & 9 & 11 & 12 & 14 & 16 & 18 & 20 \\
$\mathbf{8}$ & 11 & 12 & 14 & 16 & 18 & 20 & 22 \\
$\mathbf{9}$ & 12 & 13 & 15 & 17 & 19 & 21 & 23 \\
$\mathbf{1 0}$ & 13 & 15 & 17 & 19 & 21 & 23 & 25 \\
$\mathbf{1 1}$ & 14 & 16 & 18 & 20 & 22 & 24 & 26 \\
\hline
\end{tabular}

\begin{tabular}{c|ccccccc}
\multicolumn{7}{c}{ Transposición Lateral: niños } \\
\hline Edad & $\mathbf{P}_{\mathbf{3}}$ & $\mathbf{P}_{\mathbf{1 0}}$ & $\mathbf{P}_{\mathbf{2 5}}$ & $\mathbf{P}_{\mathbf{5 0}}$ & $\mathbf{P}_{\mathbf{7 5}}$ & $\mathbf{P}_{\mathbf{9 0}}$ & $\mathbf{P}_{\mathbf{9 7}}$ \\
\hline $\mathbf{6}$ & 8 & 9 & 11 & 13 & 15 & 17 & 19 \\
$\mathbf{7}$ & 10 & 11 & 13 & 15 & 17 & 19 & 21 \\
$\mathbf{8}$ & 11 & 13 & 15 & 17 & 19 & 21 & 23 \\
$\mathbf{9}$ & 12 & 14 & 16 & 18 & 21 & 23 & 25 \\
$\mathbf{1 0}$ & 13 & 15 & 17 & 20 & 22 & 24 & 27 \\
$\mathbf{1 1}$ & 14 & 17 & 19 & 21 & 24 & 27 & 29 \\
\hline
\end{tabular}

